\title{
Pouch failures following restorative proctocolectomy in ulcerative colitis
}

\author{
Ilona Helavirta ${ }^{1}\left(\mathbb{D} \cdot\right.$ Kirsi Lehto $^{1,2} \cdot$ Heini Huhtala $^{3} \cdot$ Marja Hyöty $^{1} \cdot$ Pekka Collin $^{1,2} \cdot$ Petri Aitola $^{2}$
}

Accepted: 17 June 2020 / Published online: 26 June 2020

(C) The Author(s) 2020

\begin{abstract}
Purpose Restorative proctocolectomy (RPC) is the most common operation in ulcerative colitis. Nevertheless, permanent ileostomy will sometimes be unavoidable. The aim was to evaluate the reasons for pouch failure and early morbidity after pouch excision.

Methods The number and the reasons for pouch failures were analysed in patients undergoing RPC 1985-2016.

Results Out of 491 RPC patients, 53 experienced pouch failure (10 women, 43 men); 52 out of 53 underwent pouch excision. The cumulative risk for excision at 5, 10 and 20 years was 5.6, 9.4 and $15.5 \%$, respectively. The reasons for failure included septic events such as fistula in $12(23 \%)$, chronic pouchitis in $11(21 \%)$ and leakage in $8(15 \%)$ patients. Functional reasons for pouch failure were recorded as poor function in $16(30 \%)$, incontinence in $12(23 \%)$ and stricture in $12(23 \%)$ patients. Multiple causes for pouch failure were recorded for individual patients. Seven cases of Crohn's disease were found among the failure cases: two before pouch excision and five after. Altogether, 15 Crohn's disease diagnoses were set in the RPC cohort, giving a percentage of $47 \%$ of pouch failure in this disorder. A complication occurred in 23 (44\%) patients within 30 days after surgery; 16 were mild (Clavien-Dindo grades I-II).

Conclusions Eleven percent of RPC patients suffered pouch failure: more men than women. The reasons were multiple. Crohn's disease created a risk of failure, but a half of these patients maintained the pouch. Morbidity after pouch excision was moderate, but in most cases slight.
\end{abstract}

Keywords Restorative proctocolectomy $\cdot$ Ileal pouch $\cdot$ anal anastomosis $\cdot$ IBD surgery $\cdot$ Pouch failure

\section{Introduction}

Since its introduction by Parks and Nicholls in 1978 [1], restorative proctocolectomy has become the procedure of choice in patients with ulcerative colitis (UC) requiring surgery. Despite the evolved details of the operation and centralization of the surgery to centres having the number of operations on an acceptable level, poor functional results and pouch-related

Ilona Helavirta

Ilona.helavirta@tuni.fi

1 Department of Gastroenterology and Alimentary Tract Surgery, Tampere University Hospital, Teiskontie 35, FI-33521 Tampere, Finland

2 Faculty of Medicine and Health Technology, Tampere University, P.O. Box 100, FI-33014 Tampere, Finland

3 Faculty of Social Sciences, Tampere University, FI-33014 Tampere, Finland complications may compel excision of the pouch and the construction of a permanent stoma. The long-term failure rate for RPC is reported to be $10-15 \%$ [2-4]. The most common causes of pouch failure have been pelvic sepsis, poor pouch function, pouchitis and Crohn's disease [4-6]. The factors leading to pouch excision are still poorly understood. This prompted us to explore the long-term risk of pouch failure, covering more than three decades of RPC cases in a highvolume hospital. We report the reasons for pouch failure, factors associated with the risk and the morbidity of pouch excision.

\section{Material and methods}

All consecutive patients with UC undergoing RPC at Tampere University Hospital between 1985 and 2016 were identified from the patient records using the ICD-9 and ICD-10 codes for UC and NCSP (Nordic Classification for Surgical 
Procedures) codes for the types of operations. A database to form an RPC registry was collected from patient files including details on patient history, surgical technique, postoperative morbidity and follow-up. On patients with pouch failure, we collected details on patient history, reasons for failure, treatment prior to failure and on the pouch excision procedure. Seventy-five patients had moved elsewhere in Finland. Data on the pouch excision of these patients were retrieved from the register of the National Institute of Health and Welfare (NIHW). The patients were identified by the NSCP procedure codes. A widely used prognostic model, the Charlson Comorbidity Index, was used to categorise pouch failure patients' comorbidities, a method based on the diagnostic codes [7]. Each comorbidity category had an associated weight (from 1 to 6, 6 being the most severe) based on the adjusted risk of mortality or resource use, and the sum of all the weights yields a single comorbidity score for a patient. A score of zero indicates that no comorbidities were found. The higher the score, the more likely the outcome to result in mortality or higher resource use. Complications were classified according to the Clavien-Dindo (C-D) classification, consisting of seven grades (I, II, IIIa, IIIb, IVa, IVb and V) [8]. Crohn's disease was diagnosed in endoscopy, either by pouch endoscopy or endoscopy from stomal entrance, histology, capsule imaging or magnetic resonance imaging (MRI) and clinical picture.

All pouches in our RPC registry are of J-type and the anastomosis is either handsewn or stapled. Pouch failure was defined as the need for a permanent ileostomy with or without pouch excision. Early complications were defined as occurring within 30 days of the operation.

\section{Statistical analysis}

The data were analysed using SPSS (Version 25.0 IBM SPSS Statistics for Windows, Version 25.0. Armonk, NY: IBM Corp.). For categorical variables, the results were given as frequencies and percentages, and for continuous variables as means and standard deviations or as medians. Chi-square or Fisher's exact test was used to assess differences in categorical variables. The binary logistic regression analysis was used to determine the predictors of pouch failure. Results are shown as odds ratios (ORs) with their $95 \%$ confidence intervals (CIs). The cumulative probability of pouch excision was estimated by the Kaplan-Meier method. Statistical significance was set at $p \leq 0.05$.

\section{Results}

\section{Basic}

The study group comprised 491 RPC patients: 209 women and 282 men. The median follow-up time of the RPC cohort was 11 years $(0-33)$. The data was gathered and analysed during 2017. The baseline characteristics of the patients in the RPC cohort and the pouch failure cohort are presented in Table 1. All the pouches were J-pouches and only five pouch operations were laparoscopic. Thirteen $(25 \%)$ patients were smokers at the time of pouch excision. This information was gathered from anaesthesia forms, and data were not available in two cases. The Charlson Comorbidity Index distribution among pouch failure patients was 0 in $89 \%, 1$ in $7.5 \%$ and 2 in $4 \%$ of the cases.

\section{Failure}

Altogether 53 (10.8\%) pouch failures were recorded: in 10 (4.7 $\%)$ out of 209 women and in $43(15.2 \%)$ out of 282 men OR 3.58 (95\% CI 1.75-7.37). Of these, 52 underwent pouch excision and one permanent loop stoma was built due to a leakage. The median time from the operation to the failure was 4.7 ( 0 26) years, in women $5.0(0.7-17)$ and in men $4.7(0-26)$ years. Half of the pouch failures occurred within the first 5 years and $70 \%$ within 7 years of RPC surgery (Fig. 1). The cumulative risk for pouch excision at 5 years was $5.6 \%$, at 10 years $9.4 \%$ and at 20 years $15.5 \%$. Multiple causes for pouch failure were recorded for individual patients as presented in Table 2 . Twenty-eight $(52.8 \%)$ had undergone surgery prior to pouch excision in order to maintain the pouch (Table 3).

\section{Histologic findings}

In pouch endoscopy (carried out in 47 cases) prior to excision, inflammation was seen by inspection in $55 \%$, fistula in the pouch in $4 \%$, fistula in the anastomosis $11 \%$, stricture in the longer limb in $11 \%$ and stricture in the anastomosis in $11 \%$. Pouch biopsy histology was available for 42 patients and showed mild inflammation in $29 \%$, chronic active inflammation in $55 \%$ and granulation tissue in $2.4 \%$ of the patients; in $14 \%$ the histology was interpreted to be normal. Of the 48 operation specimens, histology active inflammation was seen in 37 (77\%), fistula in three $(6.3 \%)$, abscess in three (6.3\%), stricture in two $(4.2 \%)$ and mucinotic carcinoma in one $(2.1 \%)$ and one was interpreted to be normal. In four cases, it was no longer possible to find the pathology statement and one patient died soon after the operation and the specimen was not analysed.

\section{Crohn's disease}

Altogether, 15 Crohn's disease diagnoses were set in the RPC cohort after the initial diagnosis of UC; pouch failure occurred in seven (47\%) while the frequency in UC was $9.7 \%$. In five of these seven cases, the diagnosis of Crohn's disease was made after pouch failure. The median age was 47 years (2172), and $1(14 \%)$ of these patients were female. In those with final diagnosis of UC and pouch failure, the median age was 
Table 1 Demographic, ileal pouch-anal anastomosis (IPAA), surgery related and information of the J-pouch and pouch failure cohorts

\begin{tabular}{lllll}
\hline & Pouch failure & & Pouch in place & $p$-value \\
\hline & $n=53$ & & $n=438$ & \\
& $n$ or median & $\%$ or range & $n$ or median & or range \\
Men & 43 & 81 & 239 & $(3.4)$ \\
BMI at time of IPAA, mean, (SD) & 25 & $(4.7)$ & 25 & $(18-72)$ \\
Age at time of IPAA (years), & 36 & $(18-71)$ & 37 & 56 \\
$\quad$ median (range) & & & & 45 \\
Anastomoses & 45 & 85 & 15 & 57 \\
$\quad$ Handsewn & 8 & 36 & 250 & $(2-48)$ \\
$\quad$ Stapled & 19 & $(4-42)$ & 11 & 0.001 \\
Covering stoma & 9 & &
\end{tabular}

44 years $(26-53)$, and $8(17 \%)$ were female.A closer inspection of pouch endoscopy and operation histology of these Crohn's patients revealed two longer limb strictures and pouch strictures while histology showed mainly chronic active inflammation. Postoperative histology showed inflammation in all patients. Of the eight patients with their pouch in place and a diagnosis of Crohn's disease, four (50\%) had died: one died of breast cancer, and the cause of death of the three patients remained obscure. Five of the patients were taking azathioprine and one was taking biologic drugs, and all were satisfied with their pouch function.

\section{Predisposing factors}

Although pouch failure was more common in men than in women, there were no significant gender differences in the causes of pouch failure, medication prior to failure or operations performed prior to failure. In the whole RPC cohort, there was no difference in leakage 15 $(7.7 \%)$ vs. $31(11 \%)$ or in the occurrence of pelvic sepsis,
$32(15.3 \%)$ vs. $43(15.2 \%)$ between women and men.Median BMI at the time of RPC did not differ between those retaining the pouch and those suffering pouch failure 24.2 vs. $25.0 \%$. BMI was drawn from the patients' anaesthesia forms: in 11 subjects, the information was missing data. In the pouch failure group, the BMI could not be analysed in three cases. None of the five laparoscopically treated patients had experienced pouch failure during their rather short follow-up.We divided the RPC patients into $\geq 65$ and younger and there was no difference in pouch failure for these groups 2 $(11.8 \%)$ vs. $51(10.8 \%) p=0.896$, respectively.

More early leakage occurred in failure patients than in those with functioning pouch; $15(28.3 \%)$ vs. $32(7.3 \%) p<$ 0.001 , and also pelvic sepsis, $15(28.3 \%)$ vs. $60(13.7 \%) p=$ 0.005 , respectively. In the pouch failure group, early relaparotomy was carried out on $11(20.8 \%)$ and of those with functioning pouch on $31(7.1 \%), p=0.003$. Covering stoma had been used in $19(35.8 \%)$ in the pouch failure group and $250(57.2 \%)$ patients in the pouch in place group, $p=0.003$.
Fig. 1 Time (years) from RPC to pouch excision. The majority of pouch failures occurred within 7 years of RPC

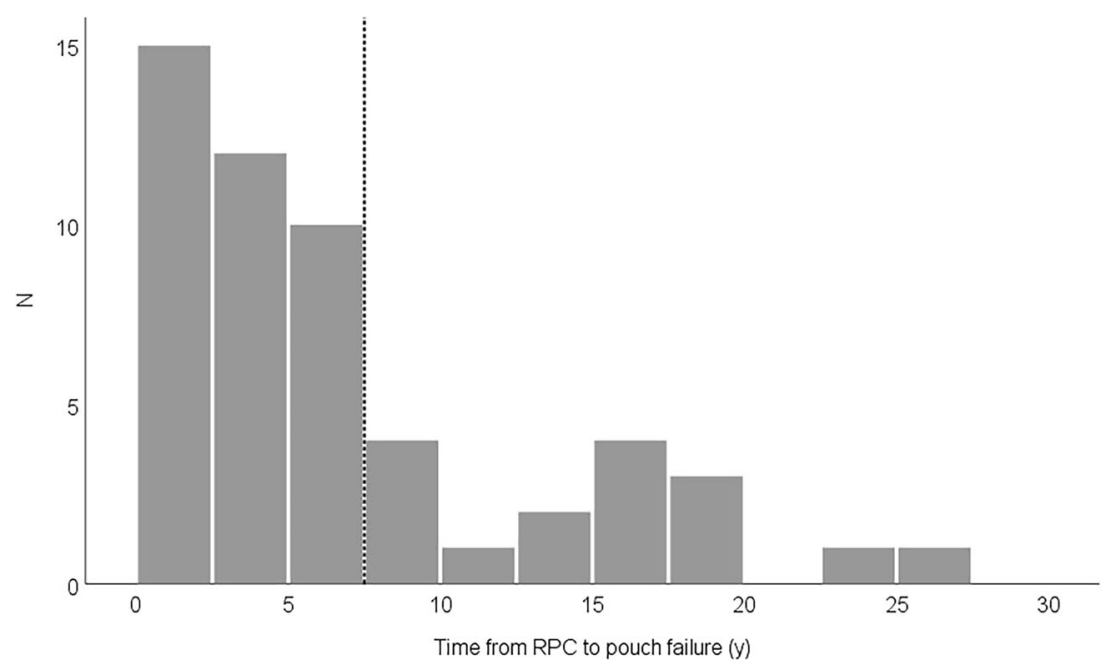


Table 2 Several reasons for pouch failure recorded for some patients

\begin{tabular}{lcc}
\hline Reason & $N$ & $\%$ \\
\hline Poor function & 16 & 30 \\
Incontinence & 12 & 23 \\
Fistula & 12 & 23 \\
Stricture & 12 & 23 \\
Chronic pouchitis & 11 & 21 \\
Leakage & 8 & 15 \\
Pouchitis & 7 & 13 \\
Crohn's disease & 7 & 13 \\
Bleeding & 1 & 2 \\
Perforation & 1 & 2 \\
\hline
\end{tabular}

The anastomoses were handsewn in $45(84.9 \%)$ and in 243 $(55.5 \%)$ patients, $p=<0.001$, respectively. The univariate and multivariate analyses of the demographic, surgery related and complication data are presented in Table 4 . The only significant parameters in the multivariate analyses were leakage, OR 3.65 (95\%CI 1.21-1.83) $p=0.022$ and male gender OR 3.83 (95\%CI $1.80-8.12) p \leq 0.001$.

\section{Operative outcome}

All pouch excision operations were performed as open surgery and median blood loss per operation was $325 \mathrm{~mL}$ (50$2300 \mathrm{~mL}$ ). Median hospital stay after pouch excision was 9 days (2-24). Readmission to hospital was recorded for 13 $(25 \%)$ of the pouch excision patients.

An early complication was recorded in 23 (44\%) of the patients after surgery but $16(70 \%)$ of these were ClavienDindo grades I-II. Early complications after pouch excision surgery are shown in Table 5. One patient died soon after pouch excision performed due to arterial bleeding at the pelvic floor; the patient declined blood transfusions for religious reasons. Clavien-Dindo grade $3 \mathrm{~b}$ complication was the cause of surgery in four (7.6\%) patients: one due to obstruction, one due to ureter damage, one stoma revision and one perineal abscess drainage.

Table 3 Prior operations aiming to prevent pouch failure

\begin{tabular}{lcc}
\hline Operation & $N$ & $\%$ \\
\hline Anastomotic dilatation & 12 & 23 \\
Loop stoma & 8 & 15 \\
End stoma & 6 & 11 \\
Fistula operation & 6 & 11 \\
Abscess drainage & 3 & 6 \\
Anastomotic repair & 2 & 4 \\
New ileoanal anastomosis & 1 & 2 \\
\hline
\end{tabular}

\section{Discussion}

This study investigated the frequency and reasons for pouch failure after RPC for UC in the second biggest IBD surgery centre in Finland in a period spanning more than three decades. The overall frequency of pouch failure was $10.8 \%$. In a previous report with a large number of patients and in a meta-analysis, similar pouch failure percentages of 9.7 [5] and $8.6 \%$ [9], respectively, were seen with a minimum follow-up time of 5 years. The Charlson Comorbidity Index among pouch failure patients was 0 in $89 \%$, which means that failure patients were otherwise mainly healthy. In a Danish national registry study with a median follow-up of 11.4 years, a 5-year risk of $9.1 \%, 10$-year risk of $12.1 \%$ and 20 -year risk of $18.2 \%$ for pouch failure were found [2].

The reasons leading to pouch failure were often multifactorial: one complication leading to another and subsequently to functional difficulties. The reasons reported in different studies may vary according to how pouch problems are categorised or recorded. The most common reasons for failure in our study were septic events in 21(40\%) (fistula 23\%, leakage $15 \%$, perforation $2 \%$ ), pouchitis in $13 \%$, chronic pouchitis in $21 \%$, Crohn's disease in $13 \%$ and functional reasons: poor function was seen in $16(30 \%)$, incontinence in $12(23 \%)$ and stricture in $12(23 \%)$ patients. In a British multicentre study comprising 94 pouch failure patients, the causes were similar: septic complications in $33(35.1 \%)$, poor function in 29 $(30.8 \%)$, pouchitis in $9(9.6 \%)$ and Crohn's disease in $5.3 \%$ [4]. Septic complications, deterioration of the functional results and Crohn's disease have also been reported earlier in 1997 [6] and in large studies in this millennium [5]. We have previously reported our functional results for those with pouch in place and $70 \%$ of the patients reported good function of the pouch as measured with the Oresland score [10]. Usually before the pouch failure, the function is devastating and the patient is too tired to consider new procedures to save the pouch.

Here, in subjects with pouch failure, the diagnosis of Crohn's disease was made before in two patients and after failure in five. Altogether, in patients with Crohn's disease diagnosed after RPC, failure was seen in 47\%. This is similar to the findings obtained in large studies $[11,12]$. No granulomas were found in our Crohn's series. A similar result was reported in the Mayo Clinic study with 35 Crohn's disease pouch failure patients; in only seven cases was pathological confirmation given [13]. In the whole RPC series, the frequency of Crohn's disease was only $3 \%$. The histologic variability of Crohn's cannot give an exact diagnosis in every case, but the diagnosis is a combination of the clinical picture, X-ray findings and histology. Nevertheless, in cases with pouch failure, the diagnosis of Crohn's disease should be borne in mind. In our series there was no significant age or gender difference for pouch failure for Crohn or UC diagnosis. 
Table 4 Univariate and multivariate analyses of demographic, surgery related and RPC complication information on RPC and pouch failure cohorts

\begin{tabular}{|c|c|c|c|c|c|c|}
\hline & \multicolumn{2}{|c|}{ Univariate } & \multirow[b]{2}{*}{$p$-value } & \multicolumn{2}{|c|}{ Multivariable } & \multirow[b]{2}{*}{$p$-value } \\
\hline & OR & $95 \% \mathrm{CI}$ & & OR & $95 \% \mathrm{CI}$ & \\
\hline Men & 3.58 & $1.75-7.31$ & $<0.001$ & 3.83 & $1.80-8.12$ & $<0.001^{*}$ \\
\hline BMI at time of RPC, mean, SD & 1.01 & $0.94-1.08$ & 0.847 & 1.01 & $0.92-1.12$ & 0.764 \\
\hline Age at time of RPC (years) median (min-max) & 1.01 & $0.99-1.03$ & 0.398 & 1.01 & $1.01-0.99$ & 0.419 \\
\hline Anastomoses & 4.51 & $2.08-9.80$ & $<0.001$ & 2.01 & $0.66-6.10$ & 0.218 \\
\hline \multicolumn{7}{|l|}{ Handsewn } \\
\hline \multicolumn{7}{|l|}{ Stapled } \\
\hline Covering stoma & 2.39 & $1.32-4.32$ & 0.004 & 0.87 & $0.41-1.83$ & 0.218 \\
\hline Pelvic sepsis & 2.49 & $1.29-4.80$ & 0.007 & & & \\
\hline Leakage & 5.01 & $2.49-10.06$ & $<0.001$ & 3.65 & $1.21-1.83$ & $0.022 *$ \\
\hline Relaparotomy after RPC & 3.44 & $1.61-7.34$ & 0.001 & 1.21 & $0.35-4.19$ & 0.764 \\
\hline Year of RPC & 0.93 & $0.90-0.96$ & $<0.001$ & 0.93 & $0.88-0.99$ & $0.013^{*}$ \\
\hline
\end{tabular}

In our study $15.2 \%$ of men and $4.7 \%$ of women suffered pouch failure. In earlier studies, there has been no significant difference in gender distribution [14-16] except in the Danish national registry study, which reported a risk in women $39 \%$ greater than that in men over time [2]. In early failures, pelvic sepsis due to anastomotic complications played a major role

Table 5 Early postoperative data after pouch excision

\begin{tabular}{lll}
\hline Surgical complication & $N$ & $\%$ \\
\hline Occlusion & 9 & 17 \\
Perineal wound infection & 3 & 6 \\
Intra-abdominal abscess & 3 & 6 \\
Bladder retention & 2 & 4 \\
Laparotomy wound infection & 2 & 4 \\
Stoma necrosis & 1 & 2 \\
Death & 1 & 2 \\
Bleeding & 1 & 2 \\
Perforation & 1 & 2 \\
Damage to ureter & 1 & 2 \\
Erectile dysfunction & 1 & 2 \\
Urinary infection & 1 & 2 \\
Bleeding from nasogastric tube & 1 & 2 \\
Sepsis & 1 & 2 \\
Clavien-Dindo classification & & \\
0 & 29 & 56 \\
1 & 10 & 19 \\
2 & 6 & 10 \\
$3 a$ & 2 & 4 \\
$3 b$ & 4 & 8 \\
5 & 1 & \\
Antibiotics postoperatively & 19 & \\
\hline
\end{tabular}

but there was no difference between genders in this report. Nor was there any difference between genders in median time from RPC to failure. We found no explanation for this gender distribution in our study.

Leakage was found to be an independent risk factor for pouch failure OR 3.65 (95\%CI 1.21-1.83) $p=0.022$. This has been reported in several reports before and the prompt diagnose and treatment of leakage cannot be stressed enough [17].

The differences in the uni- and multivariate analyses for the use of covering stoma and type of anastomoses are explained with the changes in practice through the years: handsewn anastomoses were performed mainly in 1985-2005 and covering ileostomy when considered necessary in 1985-2005 and as a routine after that. The year of IPAA has also been taken into account there and it obviously affects the follow-up time.

In our study, all but one of the pouch failure patients (52, 98\%) underwent pouch excision. In earlier reports, this percentage has been smaller: in the study by Foley $40 \%$ [18], Tulchinsky and Meagher 60\% [5, 19] and McRae 84\% [20]. In another Finnish study, all 52 failed pouches were also excised [21]. In the present study, $8(15 \%)$ out of 52 patients underwent a loop ileostomy and six patients had an end stoma built before the pouch excision operation. A pouch left in place may burden patients with incontinence. There is a need for guidelines on the management of pouch failure patients; the issues to consider are morbidity of the pouch left in place, morbidity after pouch excision, danger of dysplasia and follow-up of the redundant pouch.

In this study, morbidity of the excision was frequent but most of the complications were mild and thus acceptable. There are very few studies on morbidity after pouch excision. Karoui et al. concluded that early and especially late morbidity is high and pouch excision RPC patients should be initially 
counselled on this [22]. Prudhomme et al. studied perineal wound healing and divided the patients into groups by diagnosis. Crohn's disease seemed to be a major risk for perineal wound healing [23]. Bengtsson et al. [24] analysed their diverted pouch failure patients who did not undergo excision $(n=22)$ and found no dysplasia or cancer in histopathology in pouch biopsies; none of the patients requested further surgery and the majority had satisfactory ileostomy function. Altogether, dysplastic transformation in the ileal pouch has been estimated to be rare [25]. The present study supports this view as no dysplasia was found in the postoperative histology. One patient died of mucinotic cancer after pouch excision; this cancer was not found preoperatively despite extensive investigations.

The study was limited due to its retrospective nature and lack of systematic follow-up of the pouch failure patients.

\section{Conclusions}

Although most of the RPC patients operated on for UC had satisfactory functional outcome, $10.8 \%$ experienced pouch failure in the long run. The most common reasons for failure were deterioration of functional results after septic complications. Pouch excision is a major surgery and associated with moderate morbidity, necessitating precise planning and patient counselling. The possibility of Crohn's disease should be considered in UC patients with pouch failure who have been operated on.

Funding information This study was financially supported by the Mary and Georg Ehrnrooth Foundation and the Competitive State Research Financing of the Expert Responsibility area of Tampere University Hospital, Grant number 9P060.

Data availability The data is available upon the request from the corresponding author.

\section{Compliance with ethical standards}

Conflict of interest The authors declare that they have no conflict of interest.

Ethics approval This study was retrospective and observational. Therefore no ethical approval from the committee was required. The medical director of the study hospital granted approval. The study protocol conforms with the ethical guidelines of the 1975 Declaration of Helsinki (6th revision, 2008).

Open Access This article is licensed under a Creative Commons Attribution 4.0 International License, which permits use, sharing, adaptation, distribution and reproduction in any medium or format, as long as you give appropriate credit to the original author(s) and the source, provide a link to the Creative Commons licence, and indicate if changes were made. The images or other third party material in this article are included in the article's Creative Commons licence, unless indicated otherwise in a credit line to the material. If material is not included in the article's
Creative Commons licence and your intended use is not permitted by statutory regulation or exceeds the permitted use, you will need to obtain permission directly from the copyright holder. To view a copy of this licence, visit http://creativecommons.org/licenses/by/4.0/.

\section{References}

1. Parks AG, Nicholls RJ (1978) Proctocolectomy without ileostomy for ulcerative colitis. BMJ. 2:85-88

2. Mark-Christensen A, Erichsen R, Brandsborg S, Pachler FR, Norager CB, Johansen N, Pachler JH, Thorlacius-Ussing O, Kjaer MD, Qvist N, Preisler L, Hillingso J, Rosenberg J, Laurberg S (2018) Pouch failures following ileal pouch-anal anastomosis for ulcerative colitis. Color Dis 20:44-52

3. Wasmuth HH, Trano G, Endreseth B, Rydning A, Wibe A, Myrvold HE (2009) Long-term surgical load in patients with ileal pouch-anal anastomosis. Color Dis 11:711-718

4. Tekkis PP, Lovegrove RE, Tilney HS, Smith JJ, Sagar PM, Shorthouse AJ, Mortensen NJ, Nicholls RJ (2010) Long-term failure and function after restorative proctocolectomy - a multi-centre study of patients from the UK National Ileal Pouch Registry. Color Dis 12:433-441

5. Tulchinsky H, Hawley PR, Nicholls J (2003) Long-term failure after restorative proctocolectomy for ulcerative colitis. Ann Surg 238:229-234

6. Korsgen S, Keighley MR (1997) Causes of failure and life expectancy of the ileoanal pouch. Int J Color Dis 12:4-8

7. Charlson ME, Pompei P, Ales KL, MacKenzie CR (1987) A new method of classifying prognostic comorbidity in longitudinal studies: development and validation. J Chronic Dis 40:373-383

8. Dindo D, Demartines N, Clavien P (2004) Classification of surgical complications: a new proposal with evaluation in a cohort of 6336 patients and results of a survey. Ann Surg 240:205-213

9. Hueting WE, Buskens E, van der Tweel I, Gooszen HG, van Laarhoven CJHM (2005) Results and complications after ileal pouch anal anastomosis: a meta-analysis of 43 observational studies comprising 9,317 patients. Dig Surg 22:69-79

10. Helavirta I, Hyoty M, Huhtala H, Collin P, Aitola P (2018) Longterm functional outcome after restorative proctocolectomy: a crosssectional study. Scand J Gastroenterol 53:245-1249

11. Sagar PM, Dozois RR, Wolff BG (1996) Long-term results of ileal pouch-anal anastomosis in patients with Crohn's disease. Dis Colon Rectum 39:893-898

12. Deutsch AA, McLeod RS, Cullen J, Cohen Z (1991) Results of the pelvic-pouch procedure in patients with Crohn's disease. Dis Colon Rectum 34:475-477

13. Lightner AL, Dattani S, Dozois EJ, Moncrief SB, Pemberton JH, Mathis KL (2017) Pouch excision: indications and outcomes. Color Dis 19:912-916

14. Manilich E, Remzi FH, Fazio VW, Church JM, Kiran RP (2012) Prognostic modeling of preoperative risk factors of pouch failure. Dis Colon Rectum 55:393-399

15. Worley GHT, Patsouras D, Sahnan K, O Adegbola S, Mahmood H, Faiz OD, Clark SK (2019) Ileal pouch excision: a contemporary observational cohort. Dis Colon Rectum 62:454-462

16. Fazio VW, Tekkis PP, Remzi F, Lavery IC, Manilich E, Connor J, Preen M, Delaney CP (2003) Quantification of risk for pouch failure after ileal pouch anal anastomosis surgery. Ann Surg 238:605607

17. Worley GHT, Segal JP, Warusavitarne J, Clark SK, Faiz OD (2018) Management of early pouch-related septic complications in ulcerative colitis: a systematic review. Color Dis 20:O181-O189 
18. Foley EF, Schoetz DJJ, Roberts PL, Marcello PW, Murray JJ, Coller JA, Veidenheimer MC (1995) Rediversion after ileal pouch-anal anastomosis. Causes of failures and predictors of subsequent pouch salvage. Dis Colon Rectum 38:793-798

19. Meagher AP, Farouk R, Dozois RR, Kelly KA, Pemberton JH (1998) J ileal pouch-anal anastomosis for chronic ulcerative colitis: complications and long-term outcome in 1310 patients. Br J Surg 85:800-803

20. MacRae HM, McLeod RS, Cohen Z, O'Connor BI, Ton EN (1997) Risk factors for pelvic pouch failure. Dis Colon Rectum 40:257262

21. Lepisto A, Luukkonen P, Jarvinen HJ (2002) Cumulative failure rate of ileal pouch-anal anastomosis and quality of life after failure. Dis Colon Rectum 45:1289-1294

22. Karoui M, Cohen R, Nicholls J (2004) Results of surgical removal of the pouch after failed restorative proctocolectomy. Dis Colon Rectum 47(6):869-875
23. Prudhomme M, Dehni N, Dozois RR, Tiret E, Parc R (2006) Causes and outcomes of pouch excision after restorative proctocolectomy. BJS 93(1):82-86

24. Bengtsson J, Borjesson L, Willen R, Oresland T, Hulten L (2007) Can a failed ileal pouch anal anastomosis be left in situ? Color Dis 9:503-508

25. Borjesson L, Willen R, Haboubi N, Duff SE, Hulten L (2004) The risk of dysplasia and cancer in the ileal pouch mucosa after restorative proctocolectomy for ulcerative proctocolitis is low: a longterm term follow-up study. Color Dis 6:494-498

Publisher's note Springer Nature remains neutral with regard to jurisdictional claims in published maps and institutional affiliations. 